\title{
Expression of concern: car safety seats for children: rear facing for best protection
}

Henary B, Sherwood CP, Crandall JR, et al. Car safety seats for children: rear facing for best protection. Injury Prev 2007;13:398-402.

The manuscript 'Car safety seats for children: rear facing for best protection' was published in Injury Prevention in 2007, after peer review. The paper used US data from the National Automotive Sampling System Crashworthiness Data System to conclude that children 0-23 months were less likely to be severely injured when using a rear-facing car seat than a frontfacing car seat. This result, along with similar data from Swedish experience and biomechanical studies, has been used as the basis for public education and policy recommendations that favor a rear-facing position for children under age two in car seats.

In 2016, the journal was contacted by a biostatistician employed as an expert witness in a court case involving a car seat manufacturer. She indicated that she was unable to replicate the results of the analysis reported in the Henary et al paper. Despite requests from the editor, she did not provide details of her analysis nor did she submit a manuscript describing her analysis, her results, or their implications.

The same letter was also forwarded to authors of the Henary et al study. They, and colleagues, have communicated to the journal that their attempts to replicate the analysis also fell short. Specifically, they believe that survey weights were improperly handled in the initial analysis, which caused the apparent sample size to be larger than the actual sample size. This resulted in inflated statistical significance. It is important to stress-per the authors-there is no evidence that current recommendations are harmful. However, these field data are inadequate to statistically support the safety benefit of rear facing seats.

The journal has asked the authors to provide an erratum, correcting the analysis and results. We anticipate receiving this soon. We have also offered to publish updated analyses based on more recent data. In the meantime, we are releasing this statement of concern to alert readers and policymakers to uncertainty about the weight and significance of the findings reported herein.

Inj Prev 2017;23:e1. doi:10.1136/ip.2006.015115eoc1 